\title{
Spondyloepiphyseal dysplasia, Nishimura type
}

INSERM

\section{Source}

INSERM. (1999). Orphanet: an online rare disease and orphan drug data base.

Spondyloepiphyseal dysplasia, Nishimura type. ORPHA:163649

Spondyloepiphyseal dysplasia Nishimura type is characterized by spondyloepiphyseal

dysplasia, craniosynostosis, cataracts, cleft palate and intellectual deficit. 